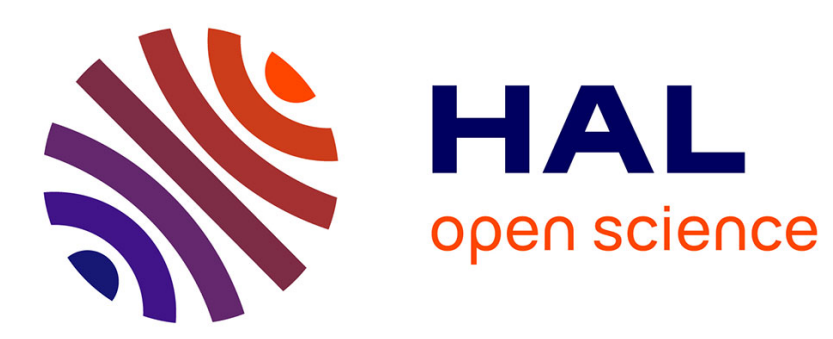

\title{
Le voyage en Patagonie revisité par Mempo Giardinelli dans Final de novela en Patagonia
}

\author{
Isabelle Bleton-Bonnet
}

\section{To cite this version:}

Isabelle Bleton-Bonnet. Le voyage en Patagonie revisité par Mempo Giardinelli dans Final de novela en Patagonia. América: cahiers du CRICCAL, 2007, 36 (1), pp.41-55. 10.3406/ameri.2007.1797. halshs-02135433

\section{HAL Id: halshs-02135433 \\ https://shs.hal.science/halshs-02135433}

Submitted on 21 May 2019

HAL is a multi-disciplinary open access archive for the deposit and dissemination of scientific research documents, whether they are published or not. The documents may come from teaching and research institutions in France or abroad, or from public or private research centers.
L'archive ouverte pluridisciplinaire HAL, est destinée au dépôt et à la diffusion de documents scientifiques de niveau recherche, publiés ou non, émanant des établissements d'enseignement et de recherche français ou étrangers, des laboratoires publics ou privés. 


\section{Le voyage en Patagonie revisité par Mempo Giardinelli dans Final de novela en Patagonia}

\section{Isabelle Bleton}

\section{Resumen}

" El viaje en Patagonia revisitado por Mempo Giardinelli en Final de novela en Patagonia ".

Con su relato de viaje publicado en 2000, Final de novela en Patagonia, Mempo Giardinelli se dedica a observar Patagonia, esa tierra de viajes y de fundaciones por antonomasia. Al narrar un viaje real durante el cual el escritor viajero intenta escribir una novela, este texto reactualiza la analogia entre el viaje y la escritura, con las inevitables errancias, rodeos y callejones sin salida que suponen ambas prácticas. El articulo se propone analizar este texto segun dos ejes : como un viaje a la vez espacial y literario, bajo forma de un texto heterogéneo que présenta simultáneamente un itinerario espacial y un verdadero laboratorio de la escritura literaria, y como un intento de doble fundación, la de un nuevo tipo de relato de viaje, y la de una nueva representación de la Patagonia, alejada del mito exotista, y que invierte la dicotomia civilización/barbarie. Revisitando y revisando el tradicional viaje de exploradores extranjeros por Patagonia, Giardinelli le da una nueva dimension, la de un viaje por lo propio y no por lo ajeno: Patagonia ya no es esa terra incognita sino el espejo de los maies contemporáneos que padece Argentina y la tierra de fundación de un posible futuro mejor para el pais.

\section{Citer ce document / Cite this document :}

Bleton Isabelle. Le voyage en Patagonie revisité par Mempo Giardinelli dans Final de novela en Patagonia. In: América : Cahiers du CRICCAL, n³6, 2007. Voyages et fondations, v2. pp. 41-55;

doi : https://doi.org/10.3406/ameri.2007.1797

https://www.persee.fr/doc/ameri_0982-9237_2007_num_36_1_1797

Fichier pdf généré le 16/04/2018 


\title{
Le voyage en Patagonie
}

\section{revisité par Mempo Giardinelli dans}

\section{Final de novela en Patagonia}

\begin{abstract}
A vec Final de novela en Patagonia', publié en 2000 , et après avoir Anavigué entre différents registres - du roman noir au roman d'introspection et d'analyse historique et culturelle, en passant par le roman de thème écologique ${ }^{2}-$, Mempo Giardinelli s'essaie à un genre nouveau dans son œuvre, le récit de voyage. Ecrit et publié immédiatement après un voyage en Patagonie effectué en compagnie d'un ami espagnol, ce récit occupe donc une place à part, ne s'inscrivant ni dans la veine du roman noir, ni dans celle du roman introspectif développée à partir de Santo oficio de la memoria. Pourtant, ce récit de voyage entretient des liens étroits avec l'œuvre fictionnelle de Giardinelli, la raison la plus apparente étant qu'il contient lui-même des fragments d'un roman que le narrateur-voyageur écrit simultanément au voyage, et qui narre également un voyage, ou plus exactement la fuite à travers la Patagonie d'un couple poursuivi par la police ${ }^{3}$. Le récit de voyage se double alors d'un récit de l'écriture, problématisant ainsi l'identité générique du texte, qui prend alors la forme d'une quête et peut être lu comme une tentative de fondation : celle d'une autre représentation de la Patagonie, qui puise dans une longue tradition littéraire tout en cherchant à $s$ 'en affranchir. En quoi Giardinelli réinvente-t-il le récit du voyage en Patagonie ? En quoi revisite-t-il le mythe de la Patagonie pour déboucher sur la fondation d'une autre représentation de cette région?
\end{abstract}

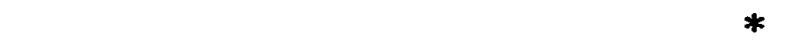

\footnotetext{
1. Mcmpo Giardinell, Final de novela en Patagonia, Barcelone, coll. " Grandes viajcros ", Edicioncs B, 2000. Le livre a ćté rćcompensé par le Prix "Grandes viajeros", décerné par les Edicioncs B ct la compagnic lbcria. Les lecteurs argentins y ont cu accès en 2001, par l'édition de poche Suma de Letras.

2. Luna caliente (1983), Madrid, Alianza Editorial, 2002 ; Qué solos se quedan los muertos, Barcclonc, Plaza y Janés, 1986 ; Santo Oficio de la memoria, Santafé de Bogotá, Editorial Norma, 1991 ; Imposible equilibrio, Barcclonc, Plancta, 1995 ; El décimo infierno, Mcxico, Colibri, 1999

3. Les deux personnages sont les héros de Imposible equilibrio. Le projet du narratcur-voyageur cst d'ecrire unc suite à ce roman.
} 
En premier lieu, il convient d'examiner les caractéristiques du récit de voyage que nous propose Giardinelli. Le texte de Final de novela en Patagonia se caractérise par une grande hétérogénéité discursive, qui a été mal comprise d'un certain public s'attendant à trouver une description exhaustive de la Patagonie et un guide de voyage, ou qui au contraire a enchanté un autre public connaisseur de l'œuvre de Giardinelli ou moins conventionnel dans ses goûts.

Certes, il peut être déroutant de trouver, au milieu d'un récit de voyage en Patagonie, un chapitre presque entièrement consacré à un portrait de Juan Rulfo, ainsi que des notes, des parties didactiques, des digressions ethnographiques, des fragments de chronique historique, un journal intime, des rêves, des poèmes, des contes, des citations en tout genre, des souvenirs personnels, des extraits d'un roman en cours d'écriture... Mais ce serait ignorer que le discours du récit de voyage a toujours été de nature hétérogène, comme le souligne Adrien Pasquali dans Le tour des horizons, critique et récits de voyages ${ }^{4}$. Il est fréquent que le récit de voyage ne se présente pas comme un discours linéaire et homogène mais comme un "montage de genres ", dont l'un des traits distinctifs serait " sa capacité à accueillir cette diversité de genres et de types discursifs, sans le souci de les homogénéiser" 5 . Le récit de voyage est fondamentalement une forme ouverte et polymorphe, un genre composite. La structure hétérogène de Final de novela... ne devrait donc guère étonner, et ferait même du texte de Giardinelli une forme canonique.

L'hétérogénéité discursive s'accompagne d'une temporalité ambiguë, elle aussi conforme au schéma narratologique classique du récit de voyage. Le récit principal hésite entre narration simultanée au présent et narration ultérieure au passé. Tantôt le narrateur donne l'illusion de relater simultanément les faits, tantôt il laisse voir la distance temporelle entre le moment des faits et le moment de la narration. Le texte oscille alors entre des notes prises sur le vif, et un livre déjà écrit. Parfois le texte est à la fois désigné comme livre et comme projet de livre : Decido que este libro, si es que va a resultar un libro de este viaje, será la ocasión propicia para hablar de ét Cette ambiguité temporelle témoigne de la refonte complexe des notes de voyage par le travail de l'écrivain. La structure même manifeste ce conflit entre deux écritures, deux styles : le journal de voyage et le livre écrit après : "La double nature - narrative et descriptive - du récit de voyage [...] révèle surtout l'ambiguïté d'un genre partagé entre les exigences souvent contradictoires de la documentation et du récit" ". En ce sens, le livre de

4. Adricn PASQuali, Le tour des horizons, critique et récits de voyages, Paris, Klincksicck, 1994, p. 107.

5. Ibid., p. 113.

6. Mempo Giardinelli, Final de novela en Patagonia, p. 62. Désormais, chaque citation de ce livre scra suivic de la page entre parcnthèses.

7. Adricn PASQuali, op. cit., p. 93. 
Giardinelli s'inscrit bien dans le modèle narratologique du récit de voyage, qui est à la fois description et narration, mimesis et diegesis, oscillant entre "le récit (presque) simultané, [...] les virtualités de l'expérience étant (presque) immédiatement consignées par le récit" et "le récit ultérieur plus ou moins fortement déterminé, et par l'issue de l'expérience passée du voyage, et par le lieu d'énonciation du récit $"$.

À présent, quelle est la fonction de cette structure hétérogène du livre? Si les digressions à fonction informative semblent couler de source, d'autres, les plus nombreuses, sont de nature littéraire et fictionnelle, et entretiennent avec le récit premier des liens plus complexes et suggestifs. Parmi ces digressions de nature littéraire, on trouve des rêves, des poèmes, des petits contes, des réélaborations de souvenirs personnels, et bien sûr le roman en cours d'écriture. Dans le cas des narrations de rêves, non seulement ils permettent de pénétrer dans l'univers intérieur du narrateur-voyageur, mais ils sont aussi des fragments d'écriture, des essais, car comme le confesse le narrateur, ils constituent une matière première littéraire, une source d'inspiration, et lui permettent une pratique quotidienne de l'écriture : Algunos de esos sueños [...] los he convertido en cuentos o utilizado como fragmentos o sueños de mis personajes en algunas novelas (p. 22). Au-delà du lien thématique qu'il peut entretenir avec le récit de voyage proprement dit, le fragment secondaire est le plus souvent offert pour lui-même, pour le plaisir de la lecture. Ainsi, les nombreux petits contes et autres narrations en tout genre, sont comme autant de voyages intérieurs, immobiles : voyages dans le temps (souvenirs), dans l'inconscient (récits de rêves), ou dans l'imaginaire (contes). C'est le voyage littéraire qui se déploie en marge du voyage réel. Giardinelli ne fait là que réinventer à sa manière la célèbre analogie écriture-voyage énoncée par Michel Butor ${ }^{9}$. Par ailleurs, sa propre conception du voyage entraîne nécessairement cette structure hétérogène :

Es imposible viajar pensando en naderías. [...] a mi me resulta imposible viajar distraídamente. Yo viajo alerta, con todos los sentidos despiertos y atentos y la memoria fluyendo como agua de manantial. Para mí es inevitable que en esta ruta vacía, en este mundo despojado y solo que es la inmensa Patagonia, me acompañen -me salven, diría yo - todos los libros que he leído. Son ellos los que imponen este ritmo pausado, medio lento, de viajar haciendo literatura de cada observación, de cada recuerdo de ese otro viaje interminable y fantástico que es la literatura universal. (p. 71)

L'auteur justifie ainsi la structure fragmentaire et hétéroclite de son récit, le recours constant à la digression, en reprenant à son compte la métaphore classique réversible, voyager=lire. Si le monde est un livre qui doit être lu/parcouru, le texte est un espace qui invite à la lecture-voyage. "La littérature est un moyen de locomotion internationale ", écrit Paul Morand dans Papiers d'identité. Le récit de voyage que nous propose Giardinelli nous 
ramène ainsi sans cesse aux textes et à l'idée que de toute manière le plus grand voyage est la littérature et que le plus grand voyageur est le lecteur.

Á cela s'ajoute le fait qu'une très grande partie des digressions qui émaillent le récit de voyage ne sont pas seulement de nature littéraire mais ont pour thème la littérature et la création littéraire. Les impressions de l'écrivain-voyageur peuvent être le point de départ d'une réflexion sur la littérature et sur son propre travail, réflexion qui se développe en marge du récit de voyage et peut par exemple prendre la forme de "méta-poèmes" traitant de la nature de la poésie et du rôle du poète.

Parfois, l'insertion d'un fragment apparaît comme problématique, comme dans le cas du long texte sur Juan Rulfo : en raison du lien a priori peu évident avec le voyage, et de son exceptionnelle longueur (le récit premier ne fait que quatre pages, alors que le "fragment" sur Rulfo en fait dix), le narrateur se sent obligé de justifier l'importance de sa digression. Au chapitre 7, intitulé Nuestra Comala patagónica, il compare Sierra Grande, ville industrielle sinistrée, presque une ville fantôme, à Comala, le village de Pedro Páramo: Mientras recorro estas mesetas, estas inmensidades de piedra y viento, y sobre todo esta noche espectral en este pueblo fantasma, siento que en cierto modo Juanito nos acompaña (p. 61). Cette analogie permet d'introduire le portrait moral de Juan Rulfo ${ }^{10}$, qu'il termine par une nouvelle explication :

De pronto temo estar defraudando la atención del lector. Pero es que estos dinamismos de la persona y el escritor que fue Juan Rulfo, hombre imperfecto y contradictorio, pero digno y ético, inexorablemente tienen que ver con la Patagonia. Los Altos de Jalisco son estas mismas sierras monumentales. Comala es esta misma Sierra Grande devastada (p. 68-69).

On l'aura compris, la véritable intention de l'écrivain-voyageur est d'établir constamment des ponts, de créer des circulations entre espace réel et espace littéraire. L'espace patagonique est appréhendé par le regard de l'écrivain-lecteur avant celui du voyageur. A plusieurs reprises, Giardinelli souligne dans son récit la nature presque fictive de ce territoire de Patagonie.

Si le livre de Giardinelli apparaît en fin de compte conforme au modèle canonique du récit de voyage ${ }^{11}$, Final de novela en Patagonia semble malgré tout le transgresser par la place importante qui est accordée à la fiction, ce qui repose la question du statut du texte. Le titre même nous le rappelle, en substituant au mot attendu de voyage l'expression fin de roman. Certes, il semblerait que ce phénomène fasse partie de la tradition, comme le remarque

10. L'autcur a ćté un ami personnel de Rulfo, qu'il a fréquenté pendant son exil au Mexique. Il le considère comme un de ses " pères" littéraires.

11. D'autres aspects du livre manifestent cettc adćquation, mais il nous semble inutile d'insister sur cette question. Signalons simplement que l'autcur opte pour la forme classique du récit de voyage, chronologique, régi par la linćarité du déplacement dans l'espace : les chapitres correspondent aux grands segments géographiques traversćs et/ou aux étapes successives du périple. Tout au long du livre, le narratcur reprend ainsi un procédé classique du récit de voyage, "l'cnfilage", qui sclon Pasquali " résulte de la successivité lićc au déplacement d'un (de plusicurs) personnage(s) "(Ibid., p. 112). 
Christine Montalbetti, qui fait le constat des emprunts constants et polymorphes de l'écriture référentielle du voyage au champ de l'écriture fictionnelle $^{12}$. Mais dans le cas du texte de Giardinelli, ce phénomène prend une toute autre ampleur, puisque parallèlement au récit du voyage proprement dit se développe la narration d'un autre voyage, fictif cette fois, effectué par un couple de personnages en fuite, et qui constitue l'ébauche d'un roman que le narrateur essaie d'écrire simultanément. Il faut préciser que pour le narrateur, la motivation principale du voyage est la recherche de conditions favorables pour terminer un roman :

Yo necesitaba despegarme de lo cotidiano para concentrarme en la novela que venía trabajando y que tenía completamente atascada, como un hueso en la garganta. Me daba vueltas en la cabeza y la verdad es que me estaba complicando la vida mucho más de lo aconsejable. Algo me decía que la Patagonia me reservaba la resolución de ese texto que yo buscaba desde hacia mucho tiempo (p. 16). ${ }^{13}$

Le roman en question est la suite de Imposible equilibrio, on y retrouve le couple protagoniste formé par Victorio et Clelia, obligés de s'enfuir à travers la Patagonie. Le thème de la fuite est cultivé par Giardinelli dans presque tous ses romans, parce que l'histoire du suspect ou du criminel en fuite est une sous-catégorie du roman noir que Giardinelli apprécie particulièrement ${ }^{14}$. Il avoue même au lecteur, après avoir narré une scène où ses deux héros échappent aux policiers :

A mí me encantan esas escenas. Si volviera a empezar, creo que hoy sería solamente un escritor de libros de aventuras, llenos de persecuciones, tiros y juramentos contra Dios y las buenas costumbres (p. 112).

Final de novela en Patagonia contient en fait deux voyages parallèles : dans la réalité, celui du narrateur et de son ami, un voyage d'agrément en Patagonie, et dans la fiction, le voyage qu'effectuent les deux personnages de fiction, Clelia et Victorio. Dans le chapitre 2, alors qu'il expose l'argument de son roman, le narrateur établit lui-même explicitement, au moyen du pronom lo qui désigne le voyage, le parallélisme entre le voyage réel à venir et celui des deux héros de roman :

En dos días llegan a la Patagonia, siempre huyendo de las policías provinciales, y asi inician un viaje que - desde luego- yo aún debía escribirlo porque nosotros mismos apenas lo estábamos comenzando (p. 28).

12. Christinc MONTAlBetrI, "Les sóductions de la fiction: enjeux ćpistémologiques", in Maric-Christinc GOMEZ-GÉRAUD ct Philippc ANTOINE (comp.), Roman et récit de voyage. Ecriture de fiction, écriture de voyage. Colloque des 2 ct 3 décembre 1999, Universitć de Picardic Jules Verne, Presses de l'Universitć de Paris-Sorbonnc, 2001, p. 99-108. Cet ouvrage ćtudic précisćment les formes et enjeux de ces mélanges constants entre récit de voyage et fiction.

13. Pour autant, ce n'est pas la sculc motivation, ce voyage en Patagonic étant aussi un vicux rêve de jeunesse que le narratcur et son ami rćalisent enfin, passće la cinquantainc : Marchábamos a una aventura que habiamos soñado todas nuestras vidas (p. 15).

14. Voir Mempo GiaRdinELl, El género negro, México, Universidad Autónoma Metropolitana, 1996, p. 53. 
Le lien étroit entre le voyage et l'écriture devient ici évident, un lien de causalité par lequel l'écriture du voyage fictif est conditionnée par le voyage réel de l'écrivain. Ce n'est pas le voyage en lui-même qui l'intéresse le plus, mais la possibilité qu'offre ce voyage de trouver l'inspiration nécessaire pour continuer et terminer l'écriture du roman : Nada me excitaba más que la perpectiva de acabar esa novela (p. 28).

L'alternance des récits des deux voyages crée peu à peu une série de parallélismes : entre les deux voyageurs réels et le couple fictionnel, qui utilisent le même véhicule (une Ford Fiesta rouge surnommée el Coloradito Pérez), et entre les deux itinéraires, pratiquement les mêmes ${ }^{15}$. Mais ce rapprochement souligne aussi des différences. Les deux voyages sont de nature fondamentalement distincte : le premier est un voyage touristique planifié, désiré, alors que le second est une fuite, imprévue et forcée. Cependant, cette proximité thématique, cette ressemblance entre les deux périples produit fatalement des interférences, des interactions. Ainsi, le voyage réel a pour effet bénéfique de débloquer l'imaginaire et de relancer l'écriture, et d'autre part, l'univers fictionnel du roman, les aventures des deux héros influencent le vécu du narrateur, qui sent la présence de ses personnages et regarde le paysage avec leurs yeux. Ceci entraîne des épisodes où les deux plans de réalité se mélangent, comme lorsque le narrateur imagine ce que feraient ses personnages dans les circonstances réelles où lui se trouve, ou alors lorsqu'il transforme en personnages de fiction des individus réellement rencontrés : Entonces se me ocurre que quizá aqui Victorio y Clelia deban usar los vínculos con otros personajes que yo he conocido en este viaje (p. 151). Il se crée une réciprocité entre les deux plans, réalité et fiction, et tout au long du livre on assiste à une écriture en direct. C'est pourquoi on pourrait qualifier le texte de véritable laboratoire de l'écriture. Le voyage réel n'est pas un but en soi, mais apparaît comme la recherche des conditions idéales pour écrire, pour fonder un roman. Sa double fonction est de mettre sous les yeux du romancier des cadres, des paysages dans lesquels vont évoluer les personnages, et de créer dans l'esprit de l'écrivain un état propice à l'écriture, une ouverture, une disponibilité, pour sortir de la stagnation. Le dépaysement, le contact avec l'autre, est une bouffée d'oxygène pour le créateur. On est face à un drôle de voyage où les paysages semblent relégués au second plan, un voyage plus intérieur que physique, plutôt un processus de création en marche, dont le dynamisme est porté par le mouvement réel dans l'espace.

Pourtant, l'écriture du roman, bien que relancée par le voyage, n'aboutira pas à une œuvre terminée et satisfaisante. La fin pose au narrateur un problème quasi insurmontable. Il propose d'ailleurs deux dénouements possibles, ce qui indique le degré d'errance dans lequel se trouve son écriture. Le premier dénouement est présenté comme le plus insatisfaisant : De hecho

15. A la fin, lcurs routes se sćparent : les voyageurs récls remontent vers le nord, le long de la cordillère, alors que les héros de fiction en fuite descendent jusqu'au fin fond de la Patagonic. 
tengo en borrador un final que yo mismo no soporto (p. 219) ${ }^{16}$. Après l'avoir écrit et inséré dans le texte, le narrateur est en proie au doute, car cette fin ratée du roman ne fait que rendre plus évidente l'impasse créative dans laquelle il se trouve : La novela me está volviendo loco, [...] y todo por la maldita cosa de que no encuentro un final que me convenza [...]. Entonces escribo, toda la tarde y toda la noche, frenéticamente (p. 224). Il en vient même à douter de la viabilité de ce projet de roman :

Mi novela, por cierto, también se está terminando, al menos en mi corazón. Aún no sé si la escribiré, ni cuándo ni cómo. Pero no ceso de redactar apuntes, notas, fragmentos. En estos días viajo demasiado ansioso y se me nota. Yo lo noto y no me aguanto. $[\ldots]$ lo que no se me quita es la incertidumbre. No sé si tengo el final de la novela. Siento que se me gana el caos. (p. 227)

Il imagine alors, dans un élan de bienveillance pour ses personnages, et dans la crainte de sombrer dans le lieu commun, un deuxième dénouement possible, moins tragique que le premier, mais qui manque de panache ${ }^{17}$ :

Empiezo a pensar que quizá la novela deba terminar de un modo menos trágico, incluso festivo, inesperado : ¿por qué no dejar que zafen del cerco? ¿qué clase de autor soy yo que no sé ayudarlos a escapar, en lugar de que terminen con el lugar común de que se matan? (p. 232)

Dans les lignes finales du livre, le narrateur affirme : El viaje por la Patagonia ha terminado y mi novela, seguro, ya tiene un final (p. 236). Mais on peut légitimement se demander si l'auteur va réellement en tirer un roman, et si cette ébauche de roman n'est pas plutôt prétexte à réfléchir sur la condition d'écrivain et sur les problèmes que pose la création, cette réflexion se développant comme un itinéraire en parallèle avec l'axe du récit de voyage.

Ce roman en cours d'écriture qui alterne avec le récit de voyage, tous ces fragments de nature et de thème littéraire, confèrent au livre un caractère hybride : à la fois récit de voyage, essai, fiction, il célèbre avant tout l'écriture comme mode de vie, comme nécessité vitale. Revenant sur sa propre expérience, en particulier l'exil, l'écrivain-voyageur explique cette relation vitale à la littérature :

16. Il s'agit d'unc fin tragique où, commc dans le film Thelma et Louise, les dcux hćros, arrivćs au bout du monde, se jettent dans le vide pour ćchapper à la policc.

17. Il imagine que ses personnages, après avoir refait leur vie au Chili, reviennent en Patagonic, tout en continuant à filer le parfait amour, ce qui a raison de la résistance cardiaque de Victorio. Le roman finit dans lc Chaco, pendant la cérémonic de crémation, avec la bande d'amis qui apparaissait dans Imposible equilibrio. 
He sido siempre un transterrado y sé lo que es esto. La transterración es un concepto que vincula al ser humano en su andar itinerante sobre la tierra. [...] Para el transterrado es el piso que pisa el que va cambiando, la base de sustentación, digamos, el lugar donde uno está parado y desde donde mira el mundo. A mi me tocó vivir una transterración forzada de casi diez años, que determinó mi existencia, la modificó y modificó también mi vida amorosa, familiar, laboral y política. Lo único inmutable fue la literatura. O mejor dicho: mi pasión literaria que $[\ldots]$ fue $[\ldots]$ otro modo de sustentación. Porque la literatura es una tierra propia, un territorio que uno lleva consigo. Quizá por la inmaterialidad de las ideas, por la levedad aparente de las palabras, la literatura es un imaginario territorio portátil que llevamos con nosotros como el caracol lleva su casa (p. 225-226).

Plus loin il définit la littérature comme el único ladrillo inmutable que hombres y mujeres tenemos para construir nuestra casa donde sea, et revisite, en la nourrissant de sa propre expérience, l'analogie entre écrire et voyager :

Escribimos emigrando, digo, escritura como movimiento y escritura en movimiento, que es como yo escribo. Escritura como el viaje que la literatura es. Escritura con la permanente nostalgia de allá cuando estoy acá, y de acá cuando estoy allá. Por eso en cualquier lugar del mundo mi única casa inmutable y permanente es el sitio en el que puedo colocar mi ordenador y escribir con la misma pasión de siempre, la de ahora, la de este instante (p. 226).

Pour Mempo Giardinelli, non seulement l'écriture est voyage, mais encore le voyage est la condition de l'écriture et donc de la littérature. Le voyage, qui n'est qu'une forme de l'exil - la transterración -, crée un état de déracinement permanent qui est la source de l'écriture, comme si l'écriture devenait le moyen de se réenraciner, à travers le texte, à travers la littérature, dans le réel. Si vivre c'est partir, voyager, quitter son port d'attache, alors écrire c'est être chez soi, se réenraciner, même en étant ailleurs.

Cette prolifération de discours autonomes ou connexes au voyage ferait presque oublier que le voyage en Patagonie existe bel et bien et, parallèlement à cette quête de l'écriture, le narrateur a aussi les yeux tournés vers l'extérieur. Mais quel est ce voyage et comment est-il effectué ?

Dans les deux premiers chapitres du livre, le narrateur explique les caractéristiques de son voyage - matériel, durée, itinéraire prévu -, ainsi que sa conception du voyage. Il a même soin de faire figurer une carte de la Patagonie qui permet au lecteur de suivre l'itinéraire des voyageurs. Cet élément paratextuel permet de postuler la vérifiabilité du récit, d'établir un pacte référentiel qui authentifie le voyage. Concernant la façon dont le narrateur conçoit le voyage, deux points semblent se dégager: d'abord le refus du tourisme conventionnel, ensuite la distance par rapport aux représentations déjà construites de la Patagonie. Autrement dit, le projet est non seulement d'inventer une nouvelle façon de voyager en Patagonie, mais aussi d'inventer un nouveau récit du voyage en Palagonie. 
Dès le début du livre, le narrateur postule et revendique une attitude anti-touristique. D'abord, il refuse tout confort. Les moyens sont modestes : véhicule ordinaire, budget limité, car, remarque-t-il : Con una camioneta $4 \mathrm{X}$ 4. mucho dinero y tiempo de sobra, cualquiera puede recorrer la Patagonia (p. 16). Ensuite, le voyage envisagé n'aura rien de ce qu'il appelle un típico y previsible recorrido turistico (p. 16). Le narrateur annonce d'emblée son rejet des formes conventionnelles et massives du tourisme patagonique, comme les excursions consistant à s'approcher le plus possible d'animaux sauvages et à les regarder vivre dans leur milieu naturel. S'identifiant aux animaux envahis par les hordes de touristes et considérant cela comme une forme de voyeurisme, il n'a pas de mots assez forts pour fustiger de telles pratiques :

No estoy de acuerdo con ninguna invasión. Eso de organizar tours para ir a ver, por ejemplo, a las elefantas a punto de parir, francamente me desagrada. [...] pienso que acaso procedo así porque a mi, como escritor y también paridor de vidas, no me gusta que se metan en lo mío cuando estoy gestando, cuando concibo. (p. 79)

Ainsi, le parcours que réalise Giardinelli va délibérément à contre-courant des itinéraires et pratiques touristiques conventionnelles. D'ailleurs, le lecteur peut avoir l'impression qu'il passe plus de temps dans sa voiture et dans les chambres d'hôtels que dehors, mais c'est parce que le vrai voyage, pour lui, est ailleurs. Avec ce livre, Giardinelli aborde le problème du voyageur contemporain qui vit à l'ère des tours-opérateurs : le vrai voyage est-il encore possible alors que tout le monde décrète " la domestication totale de la planète, la mort de l'exotisme et la généralisation du Même "18 et alors que Claude Levi-Strauss dans Tristes Tropiques, proclame " la fin des voyages"? Le voyage au sens noble, le voyage-exploration, le voyage-découverte, celui des explorateurs des XVIIIème et XIXème siècle semble appartenir définitivcment au passé. Puisque, comme le remarque le narrateur, avec un bon $4 \times 4$ et de l'argent, n'importe qui peut traverser la Patagonie, quel sens a désormais le voyage? Dans un entretien, Giardinelli explique sa démarche et revendique un autre regard sur la Patagonie :

[...] mi libro no contribuye a esa idea turística y mítica de la Patagonia. No rescata los paisajes, sino la gente. Lo importante no son las ballenas o el Glaciar Perito Moreno, sino la gente que encontré en el camino y cómo vive esa gente. Es una versión muy cruda de la realidad de la zona, que es uno de los sectores de la Argentina más marginales del país. No es un libro turístico, pero nunca va a faltar algún pelotudo que lo vea asi. ${ }^{19}$

Effectivement, le voyage de Giardinelli en Patagonie est délibérément tourné vers l'humain, vers l'aspect sociologique, et non vers les paysages. Les épisodes les plus développés sont ceux où les voyageurs rencontrent et partagent un moment de vie avec des habitants. Le lecteur est invité à décentrer le regard, à regarder non pas les paysages fameux, les sites connus,

18. Adricn Pasquali, op. cit., p. 61.

19. Mariano BlEJMAN, “ Soy un cscritor viajcro, no un viajcro que cscribc ”, Página/12, 26/1 1/2000. 
mais le néant, la monotonie, l'immensité vide, pour y découvrir les habitants, des individus anonymes mais uniques et parfois même extraordinaires. Tel est le véritable intérêt du voyage :

Al volante, reflexiono sobre lo que significa viajar y conocer ciudades que no se visitan con una perspectiva turística. Es obvio que nosotros no somos turistas previsibles y tampoco nos interesa promocionar nada. Confirmo ante mí mismo que viajo como quien camina al azar; en apariencia distraido, lo que encuentre me hará feliz, sobre todo si me abre los ojos aún más (p. 111).

L'écrivain-voyageur cherche à définir une autre façon de voyager, où c'est le hasard des rencontres qui gouverne, et non les guides touristiques, où c'est le regard du voyageur qui construit le voyage, et non le voyage (en tant qu'itinéraire prédéterminé) qui construit le regard du voyageur.

Si la fin des voyages est proclamée, du même coup, on proclame la fin du récit de voyage : "Le genre serait-il aujourd'hui autre chose qu'une survivance? S'il n'y a plus de différence, il n'y a plus de matière à récit. Ainsi finissent les grands genres... "20. Cependant, le livre de Giardinelli prouve qu'au contraire le récit de voyage non seulement survit, mais peut se renouveler, s'adapter au monde globalisé qui est le nôtre. L'invention d'un nouveau récit en Patagonie passe d'abord par une réflexion sur les représentations passées, puis par la recherche d'un nouveau langage, enfin par une relecture du mythe de la Patagonie, qui prend forme notamment à travers l'inversion de la dichotomie civilisation/barbarie.

Dans les deux premiers chapitres, le narrateur passe en revue toute la littérature liée à la Patagonie et proclame sa volonté de se débarrasser du fardeau de ces représentations. Tâche difficile, car, comme le rappelle l'auteur: [...] el Sur es para nosotros mucho más que un vacio ancestral. la Pampa y el Desierto (que es como se llamaba antiguamente a la Patagonia) son nuestra tierra literaria por antonomasia (p. 17). La Patagonie est présente dans les œuvres fondatrices de la littérature argentine, comme La cautiva d'Echeverría, Martín Fierro de J. Hernández, mais aussi dans d'autres textes classiques, récits de voyage fameux, œuvres cinématographiques ${ }^{21}$... Mais le narrateur annonce qu'il n'a pas pour intention de se laisser influencer par tous ces discours sur la Patagonie : [...] nos dimos cuenta de que tantas referencias literarias y cinematográficas no nos hacian bien. Podian incluso ser contraproducentes. Nuestro viaje debia ser el que nos saliera a nosotros, y punto (p. 23). Les indispensables représentations littéraires antérieures ne sont convoquées que pour mieux les renvoyer à leurs rayons de bibliothèque : elles encombrent le narrateur, qui souhaite ne pas les voir interférer dans sa future

20. Jcan ROUDAUT, "Quclques variables du récit de voyage ”, Nrf, $\mathrm{n}^{\circ} 377$, juin 1984, p. 69.

21. Giardinclli mentionnc: Bruce Chatwin, En Patagonia ; Roberto Arlt, Aguafuertes patagónicas : Christian Kupchik, La ruta patagónica (anthologic de textes des XVIII et XIXe s.) ; Charles Darwin, El origen dé las especies; Osvaldo Baycr, La Patagonia rebelde, ct la version cinćmatographique d'Héctor Olivera, de 1974 ; Luis Scpúlveda, Patagonia Express; ; Juan Filloy, Periplo (considćrć par l'autcur comme clase magistral de libros de viaje). 
rencontre avec la Patagonie, bien que, lucide, il ait conscience que ces textes, lus et admirés, seront toujours présents dans sa mémoire : yo ya sabia que jamás las dejaria de lado. Bien plus, ils constituent ses influences littéraires. En tant que créateur, il se pose alors le problème crucial des influences :

Para cualquier escritor las influencias son insoslayables, pero en estos tiempos hay que estar más alerta que nunca : frente al vulgar plagio que vemos todos los días, muchas veces disfrazado de "homenaje" o de "intertextualidad" cuando no es repetición textual que niega el crédito al original, se impone el desafío ético de reinventar lo conocido pero desde la creación de nuevas originalidades (p. 18-19).

Ces lignes indiquent l'un des projets du texte : répondre à un double défi, littéraire et éthique à la fois : un nouveau voyage en Patagonie, suivi d'un nouveau récit de voyage en Patagonie sont-ils possibles alors même qu'il semble qu'il n'y ait plus rien à découvrir et que tout a été écrit sur la Patagonie ? Le récit de voyage en Patagonie est pratiquement devenu un cliché : comment le revisiter sans succomber à ce cliché, tel est le défi que semble vouloir relever Final de novela en Patagonia.

Ainsi, de la recherche d'un nouveau langage pour décrire la Patagonie dépend l'originalité de la représentation. Bien que le narrateur ait recours à un certain nombre d'images traditionnelles pour nommer et décrire le paysage patagonique, il tente aussi d'inventer de nouvelles images. Parmi les images conventionnelles, on trouve celle de la Patagonie comme "fin du monde": Esa región de la Argentina que para nosotros es como un final que no se quiere ver, una especie de caida del país en el mero fin del mundo (p. 17). Ou encore, la Patagonie comme immensité, comme vide, comme néant, comme un non-paysage en somme :

Es, de hecho, una planicie de piedra, de colores ocres y amarillos, en los que la Nada comienza a ser una costumbre. La misma Nada que encontraremos durante todo el viaje, que ha desencadenado mitos y leyendas y que ejerce un enorme atractivo sobre millones de personas de todo el planeta. Curiosa, la gente. (p. 51)

Le chemin est comparé à una cinta de la nada hacia la nada, pasando por la nada (p. 141). Corrélativement, la Patagonie est un " désert", mais un désert hors du commun, presque une autre planète, elle s'apparente à un paysage lunaire. C'est une représentation préalable qui s'impose avec une telle force à l'imaginaire du narrateur qu'elle précède même sa perception, d'où le décalage entre ce qui est vu et ce qui est attendu, d'où l'impatience, la frustration, puis l'ennui face à un paysage qui tarde à correspondre à l'image mythique du désert patagonique :

El paisaje va cambiando y uno quiere que se torne lunar, porque el viajero es ansioso y necesita que lo que ven sus ojos confirme lo que él espera. [...] todavia la Patagonia no se muestra como uno la imagina: desértica, pedregosa, casi lunar. Durante un buen rato, la sensación dominante no es otra que la del tedio : la monotonia del paisaje decepciona un poco. Contrasta con la ansiedad del viajero [...] (p. 38). 
Ce mélange d'attente et d'ennui a quelque chose de paradoxal : le voyageur a une attente, celle d'être impressionné par un paysage lunaire mythique, et lorsque ce paysage lunaire s'offre interminablement à ses yeux, l'impression est la monotonie et l'ennui. Le paysage patagonique est ce paradoxe : il est à la fois vide et plein, il est ce vide qui se remplit du désir du voyageur.

À côté des images de fin du monde et de néant, et comme pour remplir ce vide d'objets, le narrateur se livre à la recherche d'images originales, par le biais de comparaisons et de métaphores surréalistes :

Estepa gigantesca, nubes inmensas como si fueran miguitas de pan del cielo, rosa gigantesca al amanecer (p. 47).

El consabido paisaje es todo piedra tras piedra y, sobre ellas, la carpeta asfáltica parece una alfombra mágica (p. 71).

Sólo el viento golpea el parabrisas del Coloradito que, se me hace, si alguien lo viera pasar desde arriba pensaría que atraviesa la inmensidad como el mercurio rojo de un termómetro sobre la piel del mundo (p. 135).

Face au glacier Perito Moreno, le narrateur, très impressionné, a tout de suite recours à la métaphore hyperbolique : les chutes d'énormes morceaux de glace dans le lac sont des fantásticos eructos de la Naturaleza. La beauté somptueuse du paysage se décline en tableaux qui défient le langage : cuadros, adonde se mire, que hasta resultan dolorosos de tan bellos (p. 150). Le canyon du Río Pinturas, impressionnant et très profond, suscite également de belles hyperboles : Una quebradura que habrá hecho Dios un dia que estaba completamente distraido (p. 198); Esto es apenas una arruga gigantesca sobre la piel del mundo (p. 199).

Le récit de voyage que nous offre Giardinelli, fruit d'une autre façon de voyager, est également porteur d'une nouvelle vision de la Patagonie, c'est pourquoi on peut parler d'un mythe revisité. Par rapport à quel mythe de la Patagonie Giardinelli effectue-t-il et écrit-il son voyage ? En premier lieu, tout discours sur la Patagonie implique la référence, explicite ou non, à la dualité civilisation-barbarie. La Patagonie est née dans l'imaginaire européen comme une incarnation de la barbarie et un vide de civilisation : une contrée sauvage peuplée d'Indiens féroces, les Patagons, aux antipodes de la civilisation. Or, Giardinelli opère un retournement sémantique : la barbarie c'est le monde occidental, le monde civilisé, présent à travers les villes et leur modernité dégradée. En effet, le narrateur, très sensible aux problématiques écologiques, constate au fil de son parcours erreurs, dysfonctionnements, absurdités et aberrations. A ce développement urbain anarchique et destructeur s'ajoute la barbarie humaine, à travers des faits criminels impunis, comme l'épouvantable fait divers dans la charmante bourgade de Los Antiguos. Cette célèbre petite ville andine proche de la frontière chilienne, fameuse pour sa production de fruits et pour son environnement somptueux, a de prime abord 
un aspect enchanteur, paradisiaque, que le narrateur décrit dans un style qui éveille les soupçons :

Enclavado en un valle cordillerano, es un pueblo rico en forestaciones, quintas y chacras verdes que después de tanta piedra dibujan para nosotros un pequeño paraíso al que da gusto entrar : una Arcadia patagónica. (p. 205)

Mais derrière les apparences se cache l'horreur et l'impunité. Lors de son séjour, le narrateur apprend une histoire épouvantable, un de ces faits divers abjects qui en disent long sur la nature humaine. Scandalisé, il souligne la coexistence dans le même lieu de la beauté et de l'ignominie :

Miro los cerezos a la vera del camino, y más allá el impresionante lago Buenos Aires. Me pregunto cómo es posible que tanta belleza contenga, en un mismo y gigantesco envase, tanta ignominia, tanto cinismo (p. 209).

Interrompant le récit du voyage, le narrateur se met à réfléchir sur le regard même du voyageur, remettant en cause sa capacité à voir la réalité : me pregunto cómo miramos los paisajes cuando viajamos, cuando somos simples $y$ desprevenidos turistas que no alcanzan a ver casi nunca, lo horroroso que está oculto (p. 209). Ce décalage entre le paysage et l'histoire cachée nous interpelle. Le voyage pose un problème éthique : comment admirer une ville, ou un pays qui protège l'impunité, comment admirer une beauté qui cache l'horreur? Un autre passage permet, de façon très significative, de mettre en évidence le renversement de la dichotomie civilisation/barbarie :

La situación en que se encuentran los indígenas patagónicos - como los de toda la Argentina, hay que confesarlo y con vergulenza - es que han sido ellos, en tanto primeros pobladores y naturales habitantes de estas tierras, los principales perjudicados por la llamada " civilización " (p. 82).

A travers ces deux exemples, le narrateur suggère que la barbarie se niche désormais au cour de la civilisation : les nouveaux barbares ne sont plus les Indiens, mais les descendants des colons, et la prétendue civilisation est dénaturée par un système économique qui les marginalise, ainsi que par la corruption et l'indolence nationale.

La Patagonie est également liée au mythe de la terre exotique. Incarnation de l'inconnu, elle est terre d'exploration et donc source de mystères. Les récits des explorateurs européens ont largement contribué à ce mythe, dont l'une des manifestations les plus frappantes est la croyance aux Indiens géants Patagons. Au XIX ${ }^{\text {ème }}$ siècle, la génération intellectuelle romantique argentine, elle-même influencée par le regard des voyageurs étrangers, n'échappait pas à la perception de la Patagonie comme terre exotique. Là aussi le texte opère une inversion. Le narrateur est un voyageur autochtone qui voyage dans son propre pays et s'adresse avant tout à ses compatriotes. Sa vision est par conséquent tout à fait dénuée d'exotisme. Le voyage en Patagonie cesse alors d'être regard sur l'altérité pour devenir regard sur le même, regard sur soi, sur son propre pays, sur ses compatriotes, d'où le discours critique et autocritique. Pourtant, en tant que chaqueño, Giardinelli 
avait des raisons de considérer la Patagonie comme exotique ${ }^{22}$, mais sa vision est avant tout celle du citoyen, et surtout de l'intellectuel, dont il a conscience du rôle essentiel, rôle qu'il définit ainsi :

[...] ser la conciencia crítica de la sociedad, reflexionar el presente y adelantar ideas para un futuro que siempre está por construirse. $Y$ otro papel que les cabe es el de ser, también, los bichos raros que los políticos y los tontos jamás saben dónde colocar ni qué hacer frente a ellos. ${ }^{23}$

Le but du narrateur est de regarder au-delà des traits exotiques, mystérieux et fascinants de la Patagonie, afin de s'en appropier, comme une partie de lui-même, de son identité nationale, et de l'intégrer à la réflexion sur son pays. Malheureusement c'est le plus souvent à travers le constat des failles et des dysfonctionnements que se réalise cette intégration. Ainsi, à propos du fait divers horrible de Los Antiguos, le narrateur constate que l'impunité est un phénomène national : Es la Argentina de la impunidad, también en la Patagonia (p. 208). Giardinelli veut aussi montrer que la Patagonie n'est pas un territoire à part, une sorte de vaste contrée encore vierge, préservée des maux de ce monde. Elle est aussi malheureusement l'Argentine, la société moderne, et on y trouve tous les maux qui affectent le pays. Il s'agit de reconnaître, au cœur de ce territoire littéraire, quasiment fictionnel, les grands problèmes contemporains de l'Argentine d'aujourd'hui. Giardinelli détruit là un mythe, celui d'une Patagonie rêvée comme terre préservée. Ainsi, le regard du narrateur-voyageur oscille entre émerveillement et critique, entre admiration et déception. La Patagonie est à la fois autre et à la fois profondément argentine. Tantôt elle transporte le narrateur dans un ailleurs, avec ses spécificités, tantôt elle le renvoit à sa propre identité d'Argentin. Le voyage cesse alors d'être un " dé-paysement " pour être un retour au pays et à ses problèmes.

On peut conclure que le récit de voyage de Giardinelli s'articule autour de plusieurs " fondations ". D'abord, Giardinelli fonde et met en pratique une autre façon de voyager en Patagonie, et comme toute fondation suppose l'inscription d'une trace - géographique, cartographique, textuelle - il réalise la fondation d'un nouveau récit de voyage. Cette fondation textuelle se double d'une autre trace, plus hésitante, qui est le roman que le narrateur tente de continuer à écrire pendant son voyage, mais qui restera une fondation

22. La Patagonic est aussi unc terra incognita pour Giardinclli, ct s'oppose au Chaco, qui est la terre natale, le licu fondatcur de son identitć, le licu de l'enfance. Un lien viscéral le rattache à cette région, comme il l'a exprimé dans quelques entrctiens et articles. Mais surtout, le Chaco constituc sa géographic littéraire, l'espace de la majorité de ses romans. Voir Mempo GIARDINELli, "El Chaco litcrario : una reinvención " in B. Potthast, K. Kohut, G. Kohlhepp (ed.), El espacio interior de América del Sur, Francfort, Vervuert Verlag, 1999, p. 413-423.

23. Maria Fernanda ABAD, cntretien avec Mempo Giardinclli, "Escritor y bicho raro ", in Nexo, la revista de El Tribuno, 14/09/2003. 
inachevée et imparfaite. En revanche, la présence de l'axe du roman introduit dans le récit de voyage une dimension réflexive et une distance du narrateur avec son propre texte, qui constamment met le récit en perspective et offre au lecteur la possibilité de partager les insatisfactions, les doutes, mais aussi les enthousiasmes et le plaisir d'écrire du narrateur. Publié à une époque charnière de la carrière de Giardinelli, une époque où il est à cheval entre deux tendances, ce livre textualise toutes les interrogations et les doutes de l'écrivain sur son propre travail, et l'amène aussi peut-être, d'une certaine manière, d'une part à en finir avec un certain pan de son œuvre, et d'autre part à entreprendre une nouvelle fondation, celle de son œuvre future. ${ }^{24}$

Á ces fondations s'ajoute celle d'une autre vision de la Patagonie, démythifiante et lucide. Quelle est cette Patagonie que Giardinelli réinvente à travers son regard et son écriture ? Elle est à la fois semblable et différente de celle des auteurs précédents : insolite et " exotique " comme celle de Chatwin, ou tragique et désespérée comme celle d'Osvaldo Bayer. Entre ces deux pôles, celle de Giardinelli se dessine, inachevée et pleine d'espoirs, et surtout, profondément argentine malgré sa marginalisation. La question sous-jacente au texte est celle-ci : la Patagonie est-elle encore cette terre promise, cette terre des possibles, cette utopie où une autre Argentine, plus responsable, plus respectueuse de l'environnement, et sachant utiliser intelligemment ses formidables ressources naturelles, serait possible ? Giardinelli a écrit ce texte un an avant la crise de 2001 , à un moment où la situation économique et sociale devenait intenable. Son voyage en Patagonie, motivé en partie par des raisons littéraires (trouver les conditions propices pour terminer un roman dont l'écriture stagnait) et personnelles (réaliser un vieux rêve de jeunesse avec un ami) est aussi une douloureuse interrogation sur l'avenir de son pays : Esta región del mundo de infinita poesia e infinita tristeza, yerma y agobiada como una madre de ubres secas. Imagen de mi adolorido país, este absurdo incomprensible (p. 54).

Isabelle BLETON-BONNET,
ENS-LSH, Lyon

24. Depuis ce livrc, Giardinclli a publić un nouveau roman à plusicurs voix, Visitas después de hora (Barcelone, Ediciones B, 2003), dans lequel il continuc, dans la lignéc de Santo oficio de la memoria, d'explorer la tendance introspective et l'univers féminin. 